\title{
Um Caso de PBL Suportado pelo Moodle no Ensino de Enfermagem
}

Giovanni Ferreira de Farias, Ed.D.-EGC/UFSC - giovanni.farias@gfarias.com

Lúcia Nazareth Amante, Dr. - NFR/UFSC - lucia.amante@ufsc.br

Fernando José Spanhol, Dr. - EGC/UFSC - fernando.spanhol@ufsc.br

Marcio Vieira de Souza, Dr. - EGC/UFSC - marciovieira@egc.ufsc.br Juliana Balbinot Reis, Dr. - NFR/UFSC - juliana.balbinot@ufsc.br

\section{Resumo}

Este relato de experiência mostra os resultados e reflexões obtidos da aplicação da metodologia Problem Based Learning (PBL) em uma disciplina de graduação em Enfermagem, na Universidade Federal de Santa Catarina. Uma turma foi dividida em dois grupos no estudo de um tópico de uma disciplina. Um destes grupos foi submetido à metodologia PBL, com trabalho em equipe, e o outro seguiu a metodologia tradicional, com aulas expositivas e estudo em duplas, sem o apoio tecnológico do Moodle. O processo foi suportado por um ambiente virtual de aprendizagem, o Moodle, para facilitar distribuição de conteúdo, comunicação virtual e avaliação por pares. Os resultados mostraram a eficácia do PBL no processo de aprendizagem e na motivação discente, enquanto revelaram algumas limitações do Moodle na mediação tecnológica.

Palavras-chave: PBL, Moodle, Enfermagem

\section{A Case of PBL Supported by Moodle in Nursing Education}

\begin{abstract}
This paper reports the results and reflections obtained from the application of the Problem Based Learning methodology in the teaching-learning process in a nursing undergraduate course at the Federal University of Santa Catarina. A class was divided into two groups in the studies of a topic of the subject matter. One of these groups was submitted to PBL methodology, with teamwork, and the other one followed the traditional methodology, with lectures and study in pairs, without the technological support by Moodle. The process was supported by a virtual learning environment, Moodle, to facilitate content distribution, virtual communication, and peer evaluation. The results showed the efficacy of the PBL in the learning process and student motivation, while revealing some limitations of Moodle in technological mediation.
\end{abstract}

Keywords: PBL, Moodle, Nursing

\section{INTRODUÇÃO}

A aprendizagem baseada em problemas, mundialmente conhecida como Problem Based Learning (PBL), é uma metodologia ativa que estimula o discente ao exercício da colaboração e ao desenvolvimento de atividades sócio interacionais enquanto realiza as atividades de aprendizado (Gomes; Rego, 2011). Ao usar a metodologia PBL, o docente abdica o papel de principal fonte de conhecimento para o discente e assume um papel de facilitador da aprendizagem. O discente, por sua vez, passa a dirigir sua aprendizagem, tendo o professor apenas como um orientador (Wood, 2003; Walker; Leary, 2009).

Como o próprio nome indica a aprendizagem com o uso de PBL se baseia em problemas que são apresentados e discutidos com o docente, fazendo um levantamento inicial dos conhecimentos necessários à solução do mesmo. Parte dos conhecimentos 
envolvidos com o problema já deve ser dominada pelos discentes, enquanto outra parte precisa ser buscada e aprendida. Com base no conhecimento que os discentes supostamente já devam ter e no conhecimento que precisam adquirir, o problema é projetado para ser aplicado na metodologia (Albanese; Michael, 1993; Woods, 2006; Thomas, 2000).

A literatura destaca algumas vantagens do uso da metodologia PBL, listadas a seguir: (Woods, 2006; Walker; Leary, 2009; Berkel et al., 2010):

1. Aprimoramento da aquisição de conhecimento multidisciplinar.

2. Maior motivação e responsabilidade para o aprendizado por parte dos discentes.

3. Aquisição das competências de auto aprendizado.

4. Aquisição de raciocínio analítico.

5. Aprimoramento da capacidade de trabalho em equipe.

Desde que foi criada em 1969, a princípio para ser implementada em cursos de medicina (Albanese; Michael, 1993; Neville, 2009), e depois disseminada em diversas instituições de ensino ao redor do mundo (Borrows, 1996), a metodologia PBL ganhou diversas adaptações, cada uma com suas peculiaridades de implementação, chamadas por Ali, Al-Dous e Samaka (2015) de "sabores da metodologia". Dentre as variantes, a que foi usada na experiência aqui descrita foi o Sete Saltos de Maastricht, criada e disseminada pela Universidade de Maastricht (Berkel et al., 2010), que se mostrou mais adequada ao contexto de aprendizagem envolvido.

\section{CONTEXTO DO PROCESSO DE APRENDIZAGEM}

Esta experiência é mais um esforço na busca de melhores práticas pedagógicas, que venham a atender a necessidade de implementar metodologias ativas de aprendizagem no ensino na área de Enfermagem, objetivando a formação de um profissional preparado para tomar decisões, liderar, se comunicar com eficiência, identificar demandas cotidianas e gerenciar serviços de saúde em um meio de trabalho multiprofissional (Pinto et al., 2016). O discente de Enfermagem precisa aprender a aprender a partir de casos realistas inerentes à sua prática profissional, trabalhando em equipe, desenvolvendo sua autonomia, responsabilidade individual e senso crítico (Zagarra; Canastra, 2014).

O contexto escolhido para experimentar a metodologia PBL foi um dos temas abordados pela disciplina $O$ Cuidado no Processo de Viver Humano II - a condição Cirúrgica de Saúde, tendo por pano de fundo o Programa Nacional de Segurança do Paciente (PNSP), o qual originou seis protocolos clínicos pelas Portarias MS no 1.377 de 09/07/2013 (Brasil, 2013a) e no 2.095, de 24/09/2013 (Brasil, 2013b). Este programa visa disseminar práticas de segurança do paciente quanto a protocolos de cirurgia segura, prática de higienização das mãos, prevenção da lesão por pressão (LP), prevenção de quedas, identificação do paciente, prescrição e administração de medicamentos.

Os docentes responsáveis pela disciplina fazem uma abordagem transversal sobre os temas envolvidos na forma de seminários e aulas, sendo o uso da metodologia PBL introduzido no segundo semestre de 2016. O foco das atividades PBL foi aplicado em um dos tópicos da disciplina, que envolve o cuidado de enfermagem para os pacientes durante o perioperatório, ou seja, desde o momento da tomada de decisão pela realização de uma cirurgia até o retorno do paciente às suas atividades normais, destacando os seguintes aspectos da atividade de enfermagem:

1. A prevenção contra lesão por pressão (LP) com uso de Escala de Braden como instrumento de avaliação da pele para prevenção deste tipo de problema. 
2. O uso da Escala de Risco de Quedas de Morse (Morse, 2009) para a avaliação de risco do paciente em período pós-operatório objetivando a prevenção de quedas e danos, além da garantia de um cuidado multiprofissional em um ambiente seguro e a promoção da educação do paciente, familiares e profissionais (Brasil, 2013c).

A conotação prática do uso das escalas de Braden e de Risco de Quedas de Morse constituiu um enfoque ideal para o aprendizado baseado em problemas, cujos descritivos foram elaborados para refletir fielmente situações comumente encontradas no ambiente profissional. O objetivo mor das atividades foi a redução de risco ao paciente e aos profissionais envolvidos e a redução da sensação de dor possível de ser realizada com o uso das escalas em questão.

Partindo-se deste cenário, foi feita a adaptação da metodologia PBL dos Sete Saltos de Maastricht com uso do ambiente virtual de aprendizagem Moodle como ferramenta de suporte. Por usar recursos online, Onan, Turan e Gürlen (2014) chamam o processo de PBL em rede (Networked PBL em inglês). Como problema a ser resolvido pelas equipes, foi concebida a descrição de casos em que o uso das escalas era necessários para sua resolução, de modo a levar os discentes a investigar a teoria, se apoderar dos conceitos envolvidos e demonstrar aptidão no uso destas escalas no trato dos pacientes envolvidos.

\section{OBJETIVOS DA PESQUISA}

Ao implementar a metodologia PBL em uma parte de uma disciplina de graduação de Enfermagem, havia três objetivos primordiais, listados a seguir.

1. Investigar a viabilidade e adequação da metodologia PBL no processo de ensinoaprendizagem na área de enfermagem.

2. Comparar os resultados de aprendizagem entre um grupo de discentes submetido à metodologia PBL com outro que seguiu a metodologia convencional expositiva do mesmo tema.

3. Investigar a adequação do Moodle como ferramenta de suporte tecnológico à metodologia $\mathrm{PBL}$, especialmente no que diz respeito à avaliação de competência em processos dos discentes pelos seus pares.

\section{A APLICAÇÃO DOS SETE SALTOS DE MAASTRICHT}

É importante mencionar que a turma envolvida no experimento, composta por 32 discentes, foi dividida em dois grupos homogêneos, com base em outras avaliações realizadas nas etapas anteriores da mesma disciplina. Metade dos discentes compôs o grupo A, que seguiu a metodologia PBL, enquanto a outra metade compôs o grupo B (grupo de controle), que seguiu a metodologia convencional expositiva, ou seja assistiu aulas teóricas convencionais, sem atividade em grupo e com exposição do mesmo caso clínico para resolução em sala de aula, logo após a exposição do tema. Uma vez que os dois grupos foram separados, passaram a ter rotina totalmente distinta, apenas voltando a ter atividade comum na avaliação do resultado de aprendizagem conceitual, no final do experimento.

O grupo A foi dividido em duas equipes de oito discentes cada, para que fosse possível ter um grupo de tamanho recomendado por Woods (2003), e cada um lidando com um caso diferente. O modelo PBL da Universidade de Maastricht (Abdalla; Gaffar, 2011), teve seu protocolo de implementação, já adaptado para a realidade pedagógica da graduação em Enfermagem da Universidade Federal de Santa Catarina, esquematizado na Figura 1 e apresentado em seguida. 

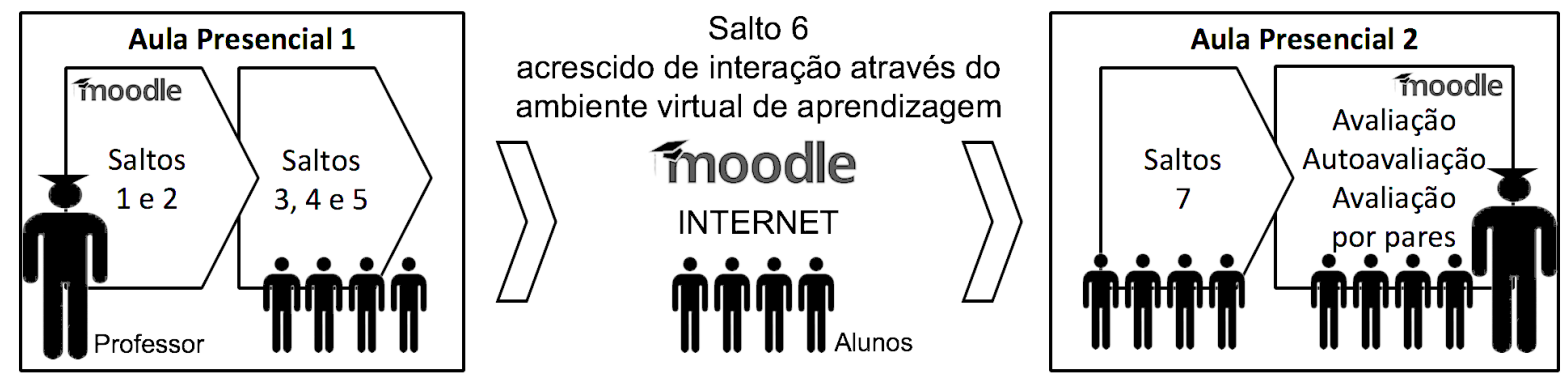

tempo

Figura 1 - Esquema de implementação dos Sete Saltos de Maastricht.

Salto 1: Esclarecer definições - O docente apresentou aos discentes a terminologia a ser utilizada no problema apresentado.

Salto 2: Apresentar o problema - O docente apresentou o problema aos discentes fazendo uso da terminologia previamente estabelecida. Neste passo o problema pôde ser estratificado, detalhado e aspectos importantes do mesmo puderam ser destacados. Apesar de nos saltos 1 e 2 ser o docente que assumiu o andamento do processo, os discentes puderam intervir para dúvidas e questionamentos. O Moodle ajudou bastante nos saltos 1 e 2, já que através dele foi disponibilizada toda a documentação referente a definições e descrição do problema, além de links para bibliografia sobre o tema.

Salto 3: Analisar o caso - Nesta fase os discentes assumiram o protagonismo do processo de aprendizagem, enquanto o docente direcionava as ações, intervindo apenas quando necessário. Cada equipe de discentes do grupo A realizou uma discussão aberta sobre o problema, tendo o docente apenas observado, feito correções de afirmações equivocadas e estimulado os mais calados a participarem do debate quando faltava iniciativa do grupo para garantir a participação de todos na discussão.

Salto 4: Reestruturar o problema - Cada equipe do grupo A teve que organizar o resultado da análise do problema, filtrando as informações relevantes para efetivação da aprendizagem e identificando o que precisava ser aprendido para resolver o problema.

Salto 5: Formular os objetivos de aprendizagem - Cada equipe do grupo A separadamente estabeleceu e documentou os objetivos de aprendizagem, traçando um plano de ação com divisão de tarefas, de modo que o esforço da equipe fosse produtivamente direcionado para resolver o problema. O docente acompanhou a discussão, fez os ajustes necessários e liberou os discentes para o trabalho.

Salto 6: Aprendizagem individual - Cada componente das duas equipes do grupo A devia agir individualmente, de acordo com o plano de ação documentado pelo relator, para realizar as pesquisas e estudos cabíveis, compartilhando o resultado com o grupo via fórum do Moodle, o ambiente virtual de aprendizagem da instituição (Moodle, 2017), algo que não faz parte da aplicação clássica de PBL, onde todos os processos de comunicação ocorrem presencialmente (Ali; Samaka; Shaban, 2011; Farias; Spanhol; Ssouza, 2016; 2017).

Salto 7: Relatório de resultados - No segundo encontro presencial, as equipes do grupo A apresentaram o que foi aprendido para os colegas de grupo, enquanto o docente fez as críticas e complementações cabíveis ao aprendizado, de modo que todos os conceitos que supostamente deveriam ser aprendidos pelos discentes fossem mencionados, discutidos e esclarecidos. As equipes usaram o Moodle para entregar relatórios de atividades e a descrição formal da solução do problema. 
Ao final da disciplina, os discentes dos grupos A (a metade da turma composta de duas equipes que seguiram a metodologia PBL) e B (a outra metade da turma que seguiu a metodologia convencional de aulas expositivas e atividade em pares para resolução do caso clínico) também foram avaliados nas competências em conceitos, ou seja, no que diz respeito ao tema que deveria ser aprendido na atividade, por meio de avaliação somativa (questionário online disponível no Moodle). Foi utilizado um questionário composto por 20 questões com diferentes níveis de complexidade, oito com transversalidade e 12 sem transversalidade. Esta avaliação envolveu todos os discentes, inclusive aqueles submetidos à abordagem convencional expositiva do tema (grupo B).

Além da avaliação sobre o resultado da aprendizagem, os discentes submetidos à metodologia PBL (grupo A) também avaliaram seus pares e fizeram autoavaliação no que diz respeito a aspectos comportamentais, relacionados com o processo de aprendizagem. As seguintes competências em processos foram avaliadas: comunicação (clareza, objetividade, escrita), colaboração (pontualidade, proatividade, relevância) e liderança (gerenciamento de tempo, compartilhamento de decisões, capacidade de motivação).

A nota final da atividade para os discentes do grupo A foi composta, portanto, pela avaliação sobre competência em conceitos (questionário) e sobre competência em processos, com 50\% de peso para cada uma delas, tal como sugerido por Woods (2006). Enquanto a nota final para os discentes do grupo B foi apenas composta pela avaliação sobre competência em conceitos (questionário).

\section{METODOLOGIA DA PESQUISA}

A metodologia de pesquisa deste experimento se baseou em uma epistemologia predominantemente interpretativista (Crotty, 2010, p. 98), envolvendo uma abordagem de pesquisa qualitativa mista explanatória sequencial e que usou o próprio Moodle como ferramenta de coleta de dados (Creswell, 2009, p. 209). A análise dos dados e conclusões da pesquisa foram baseadas em dados quantitativos obtidos por meio de escala de Likert e pela coleta de depoimentos dos discentes sobre suas impressões a respeito do processo de ensino-aprendizagem baseado na metodologia PBL. A ferramenta de coleta de dados foi o módulo Pesquisa, do Moodle, aplicado na última semana de aula apenas para os estudantes do grupo A (que seguiram a metodologia PBL).

Também foi usado o questionário de avaliação de aprendizado em competências conceituais para comparar o desempenho de aprendizado entre os grupos A (que usou abordagem PBL) e B (que usou abordagem meramente expositiva), de modo a evidenciar eventual superioridade em resultados pedagógicos de uma metodologia sobre a outra. Tal avaliação foi baseada no módulo Questionário, do Moodle, realizada presencialmente ao final da aula de encerramento da disciplina.

As questões utilizadas na coleta de pesquisa, bem como a escala Likert, são apresentadas junto aos resultados obtidos, na próxima seção.

\section{RESULTADOS OBTIDOS}

Os resultados de levantamento de perfil dos discentes do grupo A (que usaram a metodologia PBL) demonstraram não existir características que pudessem trazer algum viés às respostas dadas pelos mesmos sobre o processo pedagógico. As variáveis de perfil podem ser observadas na Figura 2. Nota-se que os discentes demonstram ter tempo suficiente para desenvolver as atividades do salto 6 , que exigem grande esforço de pesquisa e estudo, em seu tempo fora da sala de aula. Não há qualquer problema no 
trato com os recursos tecnológicos utilizados, já que todos os discentes demonstram ter média ou alta desenvoltura com tecnologia. E mais de um terço do grupo A já tiveram experiência com a metodologia PBL ou semelhante, o que facilitou a implementação da experiência.
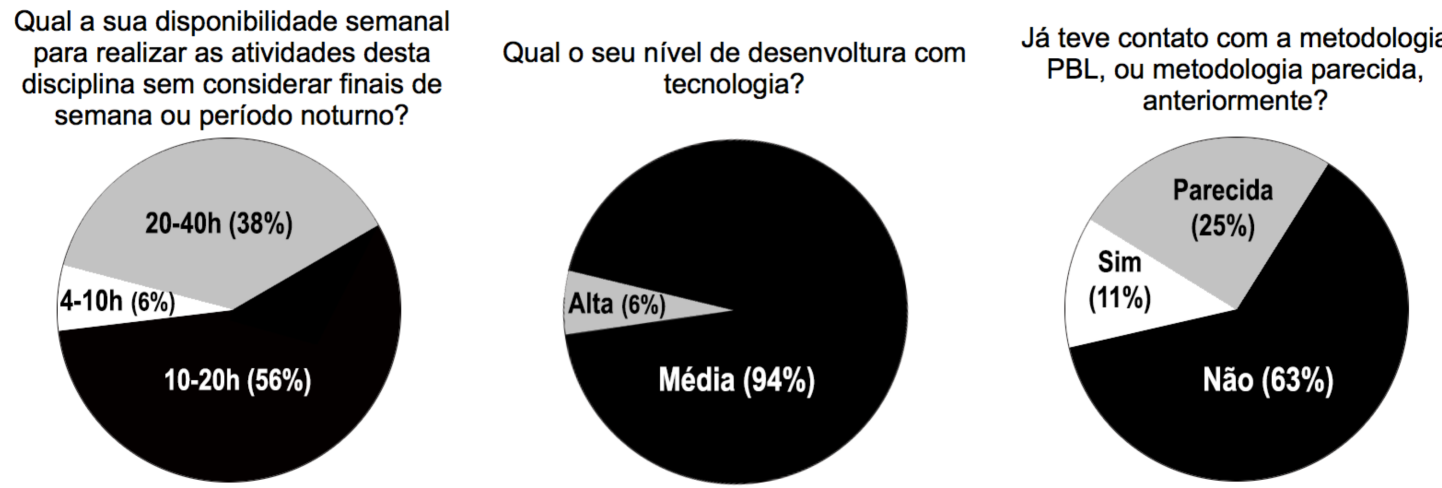

Figura 2 - Respostas referentes a perfil de cada discente.

A Figura 3 apresenta os resultados da opinião dos discentes por meio das perguntas que usaram a escala Likert (vide legenda). As diferentes cores determinam o percentual de discentes que optou por cada uma das escalas, revelando a impressão do grupo de discentes sobre o processo de ensino aprendizagem.

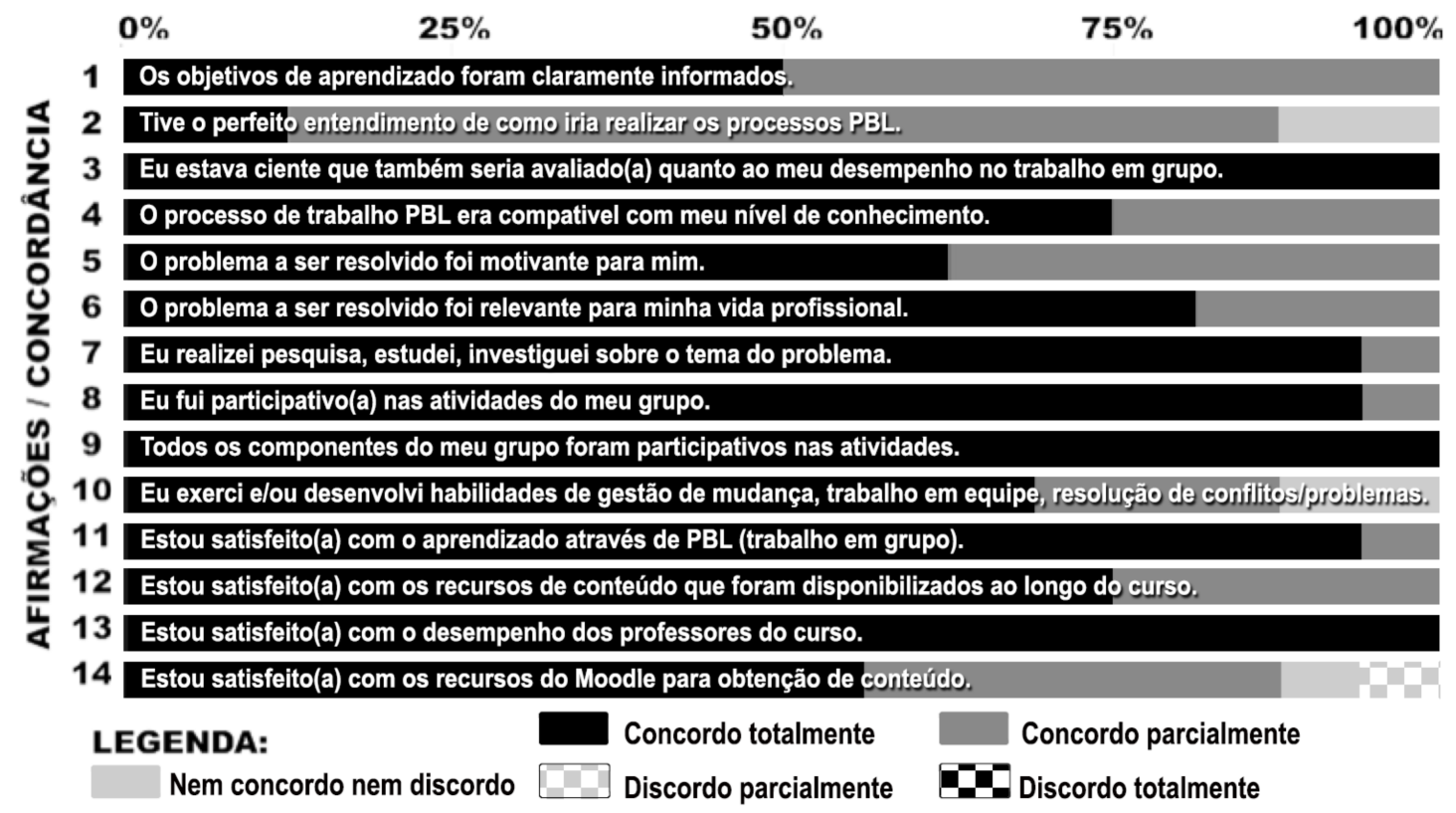

Figura 3 - Concordância com afirmações pelos discentes na implementação de PBL

Tomando como referência afirmações que obtiveram concordância plena por pelo menos $75 \%$ dos respondentes, podemos considerar que a maioria das afirmações feitas na coleta de dados via escala Likert foi satisfatória. Os discentes demonstram que estavam cientes da avaliação de competências em processos (afirmação 3), se consideravam aptos para a metodologia (afirmação 4), se sentiram motivados pela relevância do tema escolhido para sua vida profissional (afirmação 6), o que resultou em esforço de pesquisa e estudo (afirmação 7). Houve reconhecimento de participação ativa tanto pelo próprio discente quanto pelos seus pares (afirmações 8 e 9). Os discentes demonstram satisfação com o resultado de aprendizagem propiciado pela 
metodologia, pelos recursos pedagógicos disponibilizados e pela atitude dos docentes (afirmações 11, 12 e 13).

Até aqui, o que se pode concluir é o sucesso da implementação da metodologia PBL na disciplina. Porém, há afirmações que não tiveram o mesmo nível de concordância por parte dos discentes. Os discentes deixam claro que tiveram dificuldade em entender os objetivos de aprendizado (afirmação 1), que está relacionado com a abordagem inicial feito na aula 1 (vide Figura 1), bem como a documentação de instrução ao discente sobre o tema abordado, disponível no Moodle. $\mathrm{O}$ mesmo pode ser colocado como origem do fato dos discentes não terem entendido claramente sobre como ocorreria o processo PBL (afirmação 2). Algo compreensível também pelo fato da maioria não ter tido experiência prévia com a metodologia. Convém explicitar que esta também foi a primeira experiência com tal metodologia por parte dos docentes responsáveis pela disciplina, o que também contribui para equívocos na abordagem inicial sobre o processo PBL apresentado aos discentes.

O problema apresentado não foi plenamente considerado motivante pela grande maioria dos discentes (afirmação 5), o que pode ser compreendido pelo fato de que a metodologia PBL, quando sistematicamente aplicada, cobre todo o programa de uma disciplina ou mesmo de um curso de graduação. No caso, foi escolhido um tema que pôde ter sido considerado relativamente pouco desafiador aos discentes. Por outro lado, este fato também foi uma estratégia dos docentes em experimentar uma metodologia nova sem envolver um tópico mais complexo. $\mathrm{O}$ fato de ser um primeiro experimento com a metodologia motivou a falta de oportunidade de todos exercerem a liderança mencionada na afirmação 10. Em uma disciplina ou curso na qual a metodologia PBL é usada sistematicamente, há problemas a serem resolvidos a cada semana, permitindo a rotatividade de liderança e relatoria de forma mais sistemática, o que não foi o caso desta experiência.

$\mathrm{O}$ item que mais foi questionado pelos discentes é a afirmação, , referente à satisfação com os recursos do Moodle para operacionalizar a metodologia PBL. Vale salientar que o ambiente virtual utilizado foi o Moodle oficial da Universidade Federal de Santa Catarina, na sua versão 2.7, com um tema tradicional e sem integração com o Moodle App para dispositivos móveis. Todas estas características apenas dificultam o uso mais produtivo da ferramenta. Mas o que foi mais decisivo para a baixa avaliação do Moodle é a falta de ferramentas apropriadas para os processos PBL. Avaliar os pares, por exemplo, foi uma atividade realizada com o módulo Base de Dados, originalmente feito para criação de portfólios educacionais, mas que teve seu uso adaptado manualmente para permitir a coleta das avaliações dos pares.

Quanto à avaliação do aprendizado conceitual, em que se pôde comparar o resultado de aprendizagem conceitual entre os discentes dos grupos $\mathrm{A}$ e $\mathrm{B}$, os dados são reveladores. A média do grupo A foi 7,00, enquanto a média do grupo B foi 5,75 . Isso indica que os estudantes que fizeram o uso da metodologia PBL para dominar os conceitos do tema proposto demonstraram resultado $22 \%$ melhor do que os que foram submetidos à metodologia convencional na avaliação objetiva sobre os resultados de aprendizagem conceitual, realizada no final do curso.

\section{CONSIDERAÇÕES FINAIS}

Colocar em prática a metodologia PBL, antes de ser para os discentes, é estimulante para o docente. Começar a apresentação de um tema de programa de uma disciplina a partir de uma situação prática, com uma abordagem transversal e seguindo o protocolo bem definido dos Sete Saltos de Maastricht, é algo ao mesmo tempo interessante e desafiador. Não é tão fácil quanto parece ser o meticuloso planejamento 
do problema de modo a levar os discentes a se depararem com os conceitos a serem aprendidos, com um desenho de caso que não pode ser bem estruturado o suficiente para ser considerado óbvio, tão pouco mal estruturado o suficiente para resvalar em uma atividade considerada impossível de ser solucionada. Este ponto de equilíbrio também se mostra não trivial de ser ajustado quando se faz o direcionamento do referencial bibliográfico, que em composição com o caso apresentado, deve ser desafiador para o discente, mas ao mesmo tempo permitir que o mesmo chegue aos conceitos que são necessários ao aprendizado do tema.

Além do mais, apesar do grande número de publicações sobre a metodologia PBL, nenhuma das que foram lidas apresentavam uma abordagem mais prática sobre a sua implementação. Isso unido ao fato citado por Ali, Al-Dous e Samaka (2015) sobre o grande número de variantes da metodologia, torna ainda mais difícil para o docente fazer uso de PBL de forma mais assertiva. Sobram publicações com descrição superficial sobre a implementação da metodologia PBL e com maior ênfase na fundamentação teórica, enquanto faltam trabalhos publicados com maior enfoque em questões práticas de como fazer PBL. Muitos relatos de implementação PBL sequer citam a existência de avaliação de competências em processos, se limitando apenas à apresentação de caso-problema e análise de possíveis soluções (Farias; Spanhol; Souza, 2017). Um dos principais autores sobre o tema, o próprio Woods (1994, p. 2-2) causa confusão a afirmar que "estudos de caso podem ser baseados em problemas". Certamente que se tivéssemos textos com abordagem mais prática e completa sobre PBL, como o texto aqui apresentado, teria ajudado bastante nesta experiência.

Outro desafio a ser superado pelo docente é a aceitação do novo por parte dos discentes. Para quem está acostumado desde a mais tenra idade com metodologias passivas de aprendizagem, com o docente como fonte do conhecimento, em um arranjo de sala de aula que pouco mudou nos últimos dois séculos, não é fácil ser submetido a uma prática pedagógica em que é o discente que assume o protagonismo de seu aprendizado. Ainda mais quando isso ocorre no meio de um curso de nível superior, no qual a tradição da metodologia convencional expositiva está respaldada pela legislação, pelas normas internas da instituição, pelo arranjo das salas de aula e pela prática docente. Houve, portanto, resistência dos discentes para aceitarem a metodologia, ainda mais porque a experiência ocorreu em fase final de semestre letivo, quando a carga de estudos dos alunos aumenta.

Não obstante tais desafios, ao se tentar utilizar um ambiente virtual como o Moodle para ganhar produtividade nos processos, o docente descobre a insuficiência da tecnologia para tal, algo confirmado nos resultados da pesquisa. Isso é um fator muito importante, pois a experiência envolveu apenas uma parte de uma turma. A ineficácia do Moodle em dar produtividade ao processo demonstra a inviabilidade de levar a metodologia PBL a uma escala maior, diante a realidade operacional em que um docente de graduação se encontra, tal como afirmado por Ali, Samaka e Shaban (2011). Talvez por esta limitação do ambiente virtual que a grande maioria dos artigos que tratam de implementação da metodologia PBL com suporte do Moodle não inclua a parte de avaliação por pares (Farias; Spanhol; Souza, 2016), justamente o procedimento que demandou um alto grau de procedimentos manuais por parte do docente no processamento da informação da avaliação.

Apesar das dificuldades, todas superáveis com o exercício da metodologia e com o aprimoramento tecnológico, o que a experiência deixou claro é que a metodologia PBL funciona no que lhe é atribuída. Os discentes demonstram maior engajamento no processo de aprendizagem e declaram isso como fato; o discente enfoca no que se aprende (os conceitos), mas também como se aprende (os processos); há um estímulo ao 
desenvolvimento de competências no trabalho em equipe, tal como ocorre no seu futuro exercício profissional; há um ganho de autonomia no aprendizado e melhora no raciocínio clínico a partir de uma abordagem transversal e não apenas disciplinar.

A impressão que ficou para o docente ao final desta experiência é que se o discente é habituado a realizar atividades de aprendizagem com a metodologia PBL ao longo de toda sua vida discente, acaba desenvolvendo uma enorme capacidade de autonomia, responsabilidade, resiliência e de trabalho em equipe. A maior barreira a ser superada acaba sendo a cultural, em forma de acomodação, regulação e estrutura, todos corroborando para que se mantenha a aprendizagem como sempre, majoritariamente centrada no docente, em sala de aula com muitos discentes passivamente atentos à exposição dos conceitos a serem aprendidos, com pouca ou nenhuma interatividade com os colegas.

\section{REFERÊNCIAS}

ABDALLA, M.; GAFFAR A. The Seven Steps of PBL Implementation: Tutor's

Manual. 2011. Disponível em < https://goo.gl/459T5i>. Acessado em 4 agosto 2017.

ALBANESE, M.; MITCHEL, S. Problem-based learning: A review of literature on its outcomes and implementation issues. Academic Medicine, 68, 52-81. 1993.

ALI, Z.; SAMAKA, M.; SHABAN, K. A Virtual Problem Based Learning Environment in Moodle. Inted 2011: 5th International Technology, Education and Development Conference, p. 2421-2428, 2011.

ALI, Z; AL-DOUS, K. K.; SAMAKA, M. The design and delivery of hybrid PBL sessions in Moodle. International Journal of Education and Information

Technologies, v. 9, p. 105-114, 2015.

BARROWS, H. Problem-based learning in medicine and beyond: A brief overview. New Directions for Teaching and Learning 1996 (68): 3-12. 1996.

BERKEL, H; SHERPBLER, A.; HILLEN, H.; VLEUTEN, C. Lessons from Problembased Learning. New York, NY, Estados Unidos da América: Oxford University Press, 2010.

BRASIL. MINISTÉRIO DA SAÚDE. Portaria $n^{\circ}$ 1377. Aprova os Protocolos Básicos de Segurança do Paciente. Diário Oficial da União de 9 de julho de 2013a. Disponível em <http://bvsms.saude.gov.br/bvs/saudelegis/gm/2013/prt1377_09_07_2013.html.> Acesso em 30 de julho de 2017.

BRASIL. MINISTÉRIO DA SAÚDE. Portaria n o 2.095 de 24 de setembro de 2013 b. Aprova os Protocolos de Segurança do Paciente. Diário Oficial da União 25 set, 2013b. Disponível em:

$<$ http://bvsms.saude.gov.br/bvs/saudelegis/gm/2013/prt2095_24_09_2013.html>. Acesso em 30 de julho de 2017.

BRASIL. MINISTÉRIO DA SAÚDE. Agencia Nacional de Vigilância Sanitária. Fiocruz. Protocolo de Prevenção de Quedas. 2013c. Disponível:

$<$ http://www20.anvisa.gov.br/segurancadopaciente/index.php/publicacoes/item/prevenc ao-de-quedas $>$. Acesso em 30 de julho de 2017. 
CRESWELL, J. W. Research Design Qualitative, Quantitative, and Mixed Methods Approaches. Terceira ed. Los Angeles: SAGE, 2009.

CROTTY, M. The Foundations of Social Research. Londres: SAGE, 2010.

FARIAS, G. F.; SPANHOL, F. J.; SOUZA, M. V. The use of LMS to support PBL practices: A systematic review. Journal of Research \& Method in Education, v. 6, p. 3-12, 2016.

FARIAS, G. F.; SPANHOL, F. J.; SOUZA, M. V. Uma revisão narrativa sobre soluções para aprendizagem em rede baseada em problemas. In SOUZA, M. V.; STEFANI, C. (Ed.), Educação Fora da Caixa - Perspectivas e Reflexões Sobre a Inovação na Educação, Florianópolis: Perse, 2017.

GOMES, A. P.; REGO, S. Transformação da Educação Médica: É Possível Formar um Novo Médico a partir de Mudanças no Método de Ensino-Aprendizagem? Revista Brasileira De Educação Médica, v. 354, n. 354, p. 557-566, 2011.

MOODLE. Modular Object Oriented Dynamic Learning Environment. Disponível em http://www.moodle.org. Acesso em 04 de agosto de 2017.

MORSE, J. M. Preventing patient falls: establishing a fall intervention program. 2. ed. New York: Springer Pub. Co., 2009.

NEVILLE, A. Problem-Based Learning and Medical Education Forty Years on. Medical Principles and Practice, v.8, n. 1, p. 1-9. 2009.

ONAN, A.; TURAN, S.; GURLEN, E. Do Moodle Reports and Logs Meet the Needs of Educational Supervision? 9 $^{\text {th }}$ Iberian Conference on Information Systems, 2014.

PINTO, A. A. M.; MARIN, M. J. S.; TONHOM, S. F. R.; FERREIRA, M. L. S. M.. Métodos de ensino na graduação em enfermagem: uma revisão integrative da literatura. Atas CIAIQ2016. Investigação Qualitativa em Educação. Anais do $5^{\circ}$ Congresso Ibero-americano em Investigação Qualitativa. Volume 1. p. 971-980.

THOMAS, J. W. A Review of Research on Project-Based LearningThe Autodesk Foundation, 2000. Disponível em:

$<$ http://www.bobpearlman.org/BestPractices/PBL_Research.pdf $>$. Acessado em 04 de agosto de 2017.

WALKER, A.; LEARY, H. A Problem Based Learning Meta Analysis: Differences Across Problem Types, Implementation Types, Disciplines , and Assessment Levels. The Interdisciplinary Journal of Problem Based Learning. v. 3, n. 1, p. 3-24, 2009.

WOOD, D. F. ABC of learning and teaching in medicine: Problem based learning. BMJ, v. 326, n. February, p. 328-330, 2003.

WOODS, D. F. Problem-Based Learning: How to Gain the Most from PBL. Waterdown, ON, Canadá: Don Woods, 1994.

WOODS, D. R. Preparing for PBL. Waterdown, ON, Canadá: Don Woods, 2006.

ZAGARRA, G.; CANASTRA, F. O método problem based learning (PBL) no context da formação de futuros(as) enfermeiros(as). $O$ relato de uma experiência. Saúde e Desenvolvimento Humano. v. 2, n. 2; p. 105-114. 2014. 